\title{
Innovación en las regiones de Chile: una aproximación desde el análisis de sus empresas
}

\author{
Innovation in Chile's regions: An approach from the analysis \\ of their companies
}

\author{
Carmen Araneda-Guirriman ${ }^{1}$; Liliana Pedraja-Rejas ${ }^{2 *}$; Emilio Rodríguez Ponce ${ }^{3}$
}

\section{RESUMEN}

La investigación tiene por objetivo describir las relaciones entre los procesos de innovación llevados a cabo por las empresas en las regiones de Chile y factores como el Producto Interno Bruto regional, el gasto en Investigación y Desarrollo (I+D) efectuado por las instituciones de educación superior, y la estructura I+D que poseen las empresas analizadas. Para este efecto se trabaja con los resultados de la Octava Encuesta de Innovación en Empresas que da cuenta del periodo 2011-2012 y la Tercera Encuesta Nacional sobre Gasto y Personal en Investigación y Desarrollo 2011-2012, ambas realizadas por el Ministerio de Economía, Fomento y Turismo de Chile. La información fue procesada mediante análisis de correlaciones y análisis de regresión múltiple. A nivel bivariado, los resultados del estudio demuestran que existe una relación significativa entre las empresas que realizan innovación de producto y proceso con el PIB regional. A nivel multivariado, se observa que la estructura de I+D que tienen las empresas junto con el gasto en actividades I+D ejecutadas por instituciones de Educación superior resultan determinantes para las empresas que realizan innovaciones de proceso y de producto en las regiones de Chile.

Palabras claves: innovación, empresas, regiones, universidad.

\section{ABSTRACT}

This research aims to describe the relationship between innovation processes carried out by companies in the regions of Chile and other factors such as regional Gross Domestic Product (GDP), spending on research and development $(R \& D)$ carried out by Higher Education institutions, and $R \& D$ structure that own the companies analyzed. To this end the study worked with the results of the Eighth Business Innovation Survey approach for the period 2011-2012 and the Third National Survey of Expenditures and Personnel Research and Development 2011-2012, both surveys conducted by the Ministry of Economy, Development and Tourism of Chile. The data was processed by correlation and multiple regression analysis. At the bivariate level, the results demonstrate a significant relationship between the companies that make product and process innovation to regional GDP. Multivariate analysis shows that the structure of $R \& D$ that companies have; coupled with the spending on $R \& D$ activities carried out by Higher Education institutions are decisive to companies that perform process innovation and product in the regions of Chile.

Key words: innovation, companies, regions, university.

\section{Introducción}

La ciencia, la tecnología y la innovación se han convertido en un elemento central al momento de transitar a la sociedad del conocimiento (Cancino, 2009). El conocimiento y la innovación se configuran hoy como dos elementos consustanciales al desarrollo de las sociedades actuales, debido a que el crecimiento económico de una nación depende cada vez menos de la acumulación de los factores tradicionales, asociándose más a las capacidades de generar y aplicar nuevos conocimientos, ello ha derivado en la noción de las economías basadas en el conocimiento (Fernández de Lucio et al., 2011).

En este contexto, las personas y su conocimiento juegan un papel fundamental en la transición de una economía industrial hacia una basada en el conocimiento. Debido a que el desarrollo de los territorios no puede competir

\footnotetext{
Escuela de Ingeniería Industrial Informática y Sistemas, Universidad de Tarapacá. Arica-Chile.

CEUTA, Universidad de Tarapacá. Arica, Chile.

Instituto de Alta Investigación, Universidad de Tarapacá, Arica-Chile.

* Autor para correspondencia: 1pedraja@uta.cl
} 
con regiones de bajo costo, son sus industrias basadas en el conocimiento las que determinan su competitividad. En el caso del proceso de integración europea, por ejemplo, la importancia individual de los Estados decrece y el rol de la región aumenta, respectivamente.

En este sentido, cobra relevancia hablar de los sistemas de innovación, que pueden ser definidos de diferentes maneras, ya que pueden ser nacionales (Freeman, 1988), regionales (Cooke, 2008), sectoriales (Malerba, 2005) o tecnológicos (Carlsson y Stankiewicz, 1991), que involucran la creación, difusión y uso del conocimiento, considerando además componentes (universidades, empresas, institutos de investigación), relaciones entre estos y sus características o atributos (Carlsson et al., 1999). Estas estructuras surgen como concepto de política a mediados de la década de los 80 , cuyo análisis permite analizar las transiciones de sustentabilidad, entregando herramientas para identificar las debilidades del sistema (Jacobsson y Bergek, 2011). Las universidades, por consiguiente, tienen el relevante rol de contribuir en las capacidades de un sistema de innovación, no solo con la creación de ideas que puedan ser comercializadas, sino que también deben involucrarse en el proceso de transferencia de ideas de investigación hacia el éxito comercial (Veugelers et al., 2012). En efecto, las empresas emprendedoras y los recursos desarrollados en conjunto forman estos sistemas, capturándose así la naturaleza interdependiente del proceso (Feldman, 2014).

Los sistemas regionales de innovación son considerados como estructuras complejas, en donde sus componentes están dependiendo decididamente unos con otros (Hajek et al., 2014). De este modo, es importante consignar que al momento de abordar el análisis de la innovación en las regiones de Chile se debe considerar que este es un fenómeno sistémico, que involucra diversos actores, recursos y relaciones que deben ser consideradas.

En consecuencia, y con el fin de contribuir al acervo de conocimientos, el objetivo de esta investigación es describir el impacto de la presencia de estructura de I+D en las empresas y el gasto en I+D que ejecutan las instituciones de educación superior acerca de las innovaciones realizadas, de proceso y producto, por las empresas en las regiones de Chile. De esta forma se pretende generar evidencia sobre el funcionamiento de los procesos de innovación en las regiones de Chile.

\section{Marco Referencial}

\section{Innovación}

La innovación puede ser entendida como la introducción de un nuevo o significativamente mejorado producto, proceso, método de comercialización u organizativa en las prácticas de la empresa, la organización del lugar de trabajo o los vínculos exteriores (OCDE, 2005). Del mismo modo, este concepto puede ser definido como un proceso en donde las empresas dominan y ponen dentro de la práctica el diseño de productos y procesos de fabricación (Nelson y Rosenberg, 1993). En este sentido, puede ser considerada como un nuevo proceso, donde el conocimiento o las incipientes combinaciones de antiguos conocimientos son incorporados en productos y procesos de producción y posiblemente introducidos dentro de la economía. En efecto, la innovación puede ser considerada el resultado de un proceso de aprendizaje, en donde el nuevo conocimiento es conducido a las firmas con el fin de ser usado en el mejoramiento de productos y procesos de producción (Dankbaar y Cobbenhagen, 2000). Por consiguiente, se puede considerar como un proceso gradual e interactivo que se basa en la comunicación e intercambio de conocimiento, donde las organizaciones y los individuos mejoran sus formas de interacción y acumulan conocimiento.

Al momento de abordar el análisis y definición de la innovación se debe hacer referencia a Schumpeter (1927), quien la define como un cambio en las combinaciones de los factores de producción que no pueden ser efectuados por pasos infinitamente pequeños o variaciones en los márgenes. Consiste en cambios en los métodos de producción y transporte, o modificaciones en la organización industrial, o en la producción de un nuevo artículo o en la apertura de nuevos mercados o recursos materiales. La lógica schumpeteriana sugiere que los ciclos económicos son consecuencia de la innovación, pero también que las actividades y las organizaciones innovadoras son reorganizadas por las crisis económicas (Archibugi et al., 2011).

Asimismo, se ha atribuido un rol relevante a la Investigación y Desarrollo en el crecimiento económico (Romer, 1990). No obstante lo anterior, el crecimiento de los países de América Latina y el Caribe, durante la última década, fue muy moderado, a pesar de la coyuntura económica que resultó ser favorable. En efecto, los deficientes resultados de 
América Latina pueden entenderse mejor al analizar y comparar los esfuerzos I+D que se realiza con el de otras regiones, donde para el periodo 1990-1999 los países escandinavos destinaban 2,71\% del PIB a gasto real en $\mathrm{I}+\mathrm{D}$, mientras que América Latina solo gastaba 0,52\% (Álvarez et al., 2011). Se precisa que en general la inversión en la investigación y desarrollo es calculada como porcentajes del Producto Interno Bruto (Capello, 2014), y por lo general las inversiones que se efectúan en innovación son hipotéticamente relacionadas de forma positiva con el crecimiento económico (Moreno et al., 2006).

\section{Factores que inciden en el proceso de innovación}

Como se señalaba anteriormente, para analizar la innovación es importante tener en consideración las características sistémicas que tiene este proceso al constituir un sistema de innovación, este está compuesto por varios elementos (empresas privadas, universidades, centros de investigación y el gobierno, entre otros) y la relación entre estos y las instituciones (Radosevic y Kriaucioniene, 2014).

En este orden, las instituciones de educación superior se configuran como uno de los conductores más importantes de los sistemas nacionales de innovación, debido a que se requiere del sistema educacional para abarcar las tres funciones del conocimiento: la generación, la difusión y la utilización de este. Por ende, resulta importante que los sistemas de investigación y desarrollo sean orientados hacia el sistema educativo. De este modo, en una perspectiva a largo plazo, se debiera considerar como un desarrollo positivo que mejorará el rol de las universidades como generador y difusor de conocimientos (Padilla-Pérez y Gaudin, 2014).

En efecto, hay muchos factores nacionales que afectan las actividades de innovación y sus procesos de aprendizajes, dentro de ellos se encuentran la naturaleza de las instituciones científicas y tecnológicas, el sistema educacional, el sistema financiero, la estructura del mercado laboral y la especialización industrial (Filippetti y Archibugi, 2011). Del mismo modo, diferentes niveles de educación y formación y sistemas de la fuerza laboral pueden, en conjunto, caracterizar la función del mercado al generar diversos patrones de recuperación, debido a que los trabajadores pueden ser fácilmente transferidos a los crecientes sectores de la economía (OECD, 2009). En consecuencia, los polos de conocimiento pueden constituir ciudades intensivas en conocimiento y tecnología, concentrando además un alto PIB per cápita. A este respecto, se puede mencionar que el crecimiento económico de las regiones europeas está significativamente relacionado con su conocimiento y la dotación de su capital humano (Hajek et al., 2014).

A modo de ejemplo se puede señalar que durante el 2012 en México se realizó un estudio con el fin de conocer los factores que inciden en la capacidad de innovar de las economías mundiales, utilizando para este fin datos del Global Competitiveness Report del periodo 2001-2009 (excepto el 2005) de 78 países. Los resultados de este estudio indican que el capital de riesgo y la calidad del sistema educativo en los países son los factores que tienen mayor incidencia en la capacidad de innovar de un país. A esto le siguen la inversión en I+D por parte de las empresas y la disponibilidad de científicos e ingenieros. Es destacable que en este estudio no resultó significativo el PIB per cápita (Santillán 2012). Complementando lo anterior, se puede señalar el caso de Australia, donde los datos de la OCDE revelan que el gasto bruto en investigación y desarrollo fue de 21 billones en el periodo 20062007, excediendo en $2 \%$ del PIB por primera vez en su historia. Asimismo, las empresas realizan una mayor cantidad de investigación y desarrollo para el mismo periodo, gasto que se ha incrementado sustancialmente desde 1980 , pero con $1,15 \%$ del PIB permanece sustancialmente bajo del promedio de la OCDE, de 1,56\%. Las universidades, a su vez, gastan $\$ 5,4$ billones en $\mathrm{I}+\mathrm{D}$, cuyo financiamiento proviene mayoritariamente del gobierno, y solo $6 \%$ de la investigación en la universidad se financia con recursos provenientes de empresas (Dodgson et al., 2011).

Del mismo modo, aspectos como la estructura y las políticas de personal tienen un rol significativo en la intensidad de la innovación al interior de la organización, como resultado de estos desafíos asumidos están las organizaciones de investigación tecnológica al interior de una red colaborativa de innovación (Albors-Garrigos et al., 2014). El rol de la estructura industrial resulta entonces crucial para la innovación regional y la puesta al día de estas, como sucede en el caso de China, donde el rol de las empresas es central para la innovación en el país, pues hay una aceptación tácita de que el sector no estatal posee un potencial de innovar 
mucho mayor que las empresas propias del Estado. En efecto, el impacto de un perfil institucional sobre la innovación puede entenderse mejor cuando se conjugan las ideas con la capacidad de absorción (Prevezer et al., 2013). Coincidiendo de esta forma con los atributos de un sistema regional de innovación, relacionados con la capacidad del territorio para generar y absorber conocimiento y la capacidad de transformar la investigación y desarrollo en innovación y crecimiento económico.

Para el entorno socioeconómico es importante considerar aspectos que reflejen las características socioeconómicas de la región, donde se incluye el PIB regional, el que puede ser considerado como el atributo del stock de conocimiento en una región y el grado de sofisticación de la demanda. En este sentido, cabe precisar que el PIB per cápita mide el estado general de desarrollo del país. Es importante considerar dentro de este sistema regional de innovación los subsistemas vinculados a la aplicación y explotación de conocimiento, es decir, las empresas, y por otro lado el subsistema asociado a la generación y difusión de conocimiento, por medio de universidades, institutos de investigación, agencias de transferencia tecnológica, cuerpos de gobierno local y regional, responsables de las políticas y prácticas que soporten la innovación (Hajek et al., 2014).

El PIB per cápita tiene una capacidad explicativa tanto para el gasto en I+D de las empresas como el de las universidades. También se puede vincular al esfuerzo en innovación, donde existe una relación positiva, debido a que los países más avanzados son los que tienen mayor capacidad innovadora (Gámir y Durá, 2010).

Las condiciones económicas, junto con los beneficios que genera la innovación en las industrias y en la sociedad serán más favorables en la medida en que se diseñen políticas públicas de investigación y desarrollo e innovación que permitan articular e integrar los actores que conforman el sistema de innovación, como lo son las universidades, con sus centros y laboratorios de investigación y desarrollo y su rol de formación de capital humano, las industrias que aplican sus conocimientos y le incorporan valor, la administración pública que define y coordina políticas industriales de innovación, las entidades financieras de los sectores privados y otros organismos vinculados a este sistema (Piñero et al., 2013).

Cabe consignar que dentro de los procesos de innovación cobra vital importancia el capital humano calificado presente en los territorios, ya que el personal con las competencias necesarias es vital para que una organización logre incrementar su capacidad innovadora. Esto es sumamente relevante, pues según un estudio que buscaba analizar la relación entre la formación académica y los resultados de innovación, se clasificaron las empresas por conglomerados utilizando para ello el nivel educativo, evidenciándose en los análisis que los resultados de innovación son mayores en el conglomerado de profesionales (Páez, 2012). En efecto, el capital humano especializado es considerado como una variable a considerar al momento de analizar la fortaleza de las funciones de un sistema de innovación tecnológica, en conjunto con las patentes, entre otros (Jacobsson y Bergek, 2011).

En el caso particular de Chile, no se visualiza un sistema articulado de innovación y producción científico-tecnológica a pesar del trabajo que realizan instituciones como CONICYT y de todos los esfuerzos que se han efectuado en esta materia (González y Espinoza, 2011). Asimismo, en Chile existe poca evidencia cuantitativa sobre la relación entre la innovación y productividad de los países en desarrollo (Álvarez et al., 2011).

Por consiguiente, se observa que Chile se encuentra distante de lo que ocurre en otros países, debido a la falta de los recursos necesarios para el desarrollo de la investigación y la innovación como actividades fundamentales de la universidad (González y Espinoza, 2011). En este sentido, cabe señalar que en Chile la innovación es financiada principalmente por el Estado (universidades, institutos de investigación, etc.) contrastando con los otros países de la OCDE, donde el gasto es financiado en más del $60 \%$ por la empresa privada, logrando de esta forma una mayor pertinencia productiva de la investigación (Cruz, 2008). Esta situación puede ser entendida como la heterogeneidad dinámica del sistema de Ciencia y Tecnología, caracterizado como la articulación y no articulación de la producción científica con los requerimientos sociales, productivos y de soluciones de innovación que se le demanda a la ciencia, lo que va generando brechas (Cancino, 2011).

En Chile, la División de Innovación del Ministerio de Economía, Fomento y Turismo tiene por finalidad coordinar las diferentes instituciones públicas relacionadas a la implementación de los planes contenidos en la Política Nacional de Innovación 2010-2014, encargándose además 
de la ejecución del Fondo de Innovación para la Competitividad, velando por el fiel cumplimiento de sus objetivos y funcionamiento y de asistir técnicamente al ministro y al subsecretario de economía en los lineamientos, decisiones, planes y programas establecidos. Es así como este Ministerio periódicamente monitorea el estado de la innovación a nivel nacional, aplicando la Encuesta de Innovación en Empresas, con el fin de obtener información respecto de la estructura del proceso de innovación de las empresas.

De acuerdo con los resultados de un estudio realizado en el sector manufacturero de Chile por Álvarez, Bravo-Ortega y Navarro (2011) se evidencia una mayor probabilidad de inversión en I+D por parte de las grandes firmas en donde es más factible además la realización de innovaciones de productos, a su vez, la intensidad del gasto en I+D aumenta la probabilidad de innovar en procesos, ello no afecta la posibilidad de innovar en productos, igualmente se observa que una menor "apropiabilidad" disminuye la opción de innovar en procesos, y que la productividad incrementa la innovación de procesos.

En consecuencia, a partir de la literatura revisada se puede establecer que la innovación es un fenómeno relevante y clave para el desarrollo de las naciones, es por tal razón que esta investigación pretende describir el impacto de la presencia de estructura de I+D en las empresas y el gasto en I+D que ejecutan las instituciones de educación superior sobre las innovaciones realizadas, de proceso y producto, por las empresas en las regiones de Chile. De esta forma se pretende generar evidencia acerca del funcionamiento de los procesos de innovación en las regiones de Chile, en qué medida el PIB regional, el gasto en I+D, el gasto en educación impactan en la innovación de las empresas de cada región.

La hipótesis a verificar es la siguiente: las innovaciones realizadas por las empresas en las regiones de Chile se ve determinada por la presencia de unidades especializadas en la investigación y desarrollo y por el gasto en investigación y desarrollo que se efectúa en la educación superior.

\section{Metodología}

La metodología de la investigación es de tipo cuantitativa, y tiene un propósito descriptivo, ya que tiene por fin describir los procesos de innovación que experimentan las regiones, por medio de sus empresas.

Como fuente de información secundaria se usaron los resultados de la Octava Encuesta de Innovación en Empresas que da cuenta del periodo 2011-2012, instrumento que sigue el modelo y lineamientos generales definidos por la OCDE y la Community Innovation Survey (CIS). También se utilizaron los resultados de la tercera encuesta nacional relativo a gasto y personal en investigación y desarrollo 2011-2012. Ambas realizadas por el Ministerio de Economía, Fomento y Turismo de Chile.

Las variables consideradas fueron obtenidas de los resultados de esta encuesta y que a continuación se indican (Tabla 1).

Tabla 1. Variables y medidas.

\begin{tabular}{lll}
\hline \multicolumn{1}{c}{ Variable } & Indicador & Fuente de información \\
\hline & $\begin{array}{l}\text { \% de gasto en I+D ejecutado por educación } \\
\text { superior 2012 }\end{array}$ & $\begin{array}{l}\text { Resultados de tercera encuesta } \\
\text { nacional relativo a gasto y } \\
\text { personal en investigación y } \\
\text { instituciones de educación superior }\end{array}$ \\
& & desarrollo 2011-2012 \\
\hline
\end{tabular}

\begin{tabular}{|c|c|c|}
\hline Empresas que realizaron innovación de producto & $\begin{array}{l}\text { Número de empresas según novedad de la } \\
\text { innovación de producto para el mercado }\end{array}$ & \multirow{3}{*}{$\begin{array}{l}\text { Resultados de la octava } \\
\text { encuesta de innovación en } \\
\text { empresas, 2011-2012 }\end{array}$} \\
\hline Empresas que realizaron innovación de proceso & $\begin{array}{l}\text { Número de empresas según novedad de la } \\
\text { innovación de proceso para el mercado }\end{array}$ & \\
\hline Estructura I+D en las empresas & $\begin{array}{l}\text { Número de empresas que poseen unidad formal, } \\
\text { departamento o laboratorio I+D según región }\end{array}$ & \\
\hline Producto Interno Bruto (PIB) regional & PIB per cápita regional 2012 & $\begin{array}{l}\text { Cuentas nacionales de Chile, } \\
\text { PIB regional 2012, Banco } \\
\text { Central de Chile }\end{array}$ \\
\hline
\end{tabular}

Fuente: Elaboración propia. 
Se señala que previo al procesamiento de los datos se creó la variable "empresas que realizan innovación en la región", calculada a partir del promedio de las variables "empresas que realizaron innovación de proceso" y "empresas que realizaron innovación de producto".

Para el procesamiento de la información en primera instancia se efectuó un análisis bivariado por medio de un análisis de correlaciones. Posteriormente se realizó una regresión lineal múltiple con la siguiente ecuación de regresión, con el fin de determinar el impacto en el desarrollo de innovaciones de las empresas:

$$
\mathrm{V}_{\mathrm{x}}=\beta_{0}+\mathrm{a} \beta_{1}+\mathrm{b} \beta_{2}+\varepsilon_{\mathrm{i}}
$$

Donde $\mathrm{V}_{\mathrm{x}}$ es la variable dependiente, es decir, las empresas que realizan innovaciones de proceso y de producto; $\beta_{0}$ constante del modelo; a es la variable independiente denominada gasto de I+D de actividades ejecutadas por instituciones de educación superior; $\beta_{1}$ factor de ponderación; $\mathrm{b}$ variable independiente estructura $\mathrm{I}+\mathrm{D}$ en las empresas; $\beta_{2}$ factor de ponderación; y ei: error residual del modelo.

\section{Resultados y Discusión}

La Tabla 2 muestra las correlaciones de Pearson con las relaciones bivariadas entre las variables mencionadas.

Se indica que la variable empresas que realizan innovación de proceso y de producto por región se correlaciona estrechamente con el PIB per cápita regional, lo que es coincidente con la literatura que señala que los procesos de innovación en los territorios tienen un impacto positivo en el crecimiento económico de este. En efecto, como parte crucial del proceso de innovación se considera a la difusión de conocimiento, siendo este un factor clave para lograr ventaja competitiva, debido a que la innovación hace productos más competitivos y que por ende ingresan a más mercados (Galindo y Méndez, 2014). De igual forma, la presencia de una estructura de apoyo de la innovación, como lo son las unidades, departamentos y laboratorios de I+D en las empresas es significativa para la innovación desarrollada en estas. Reforzándose de este modo la relevancia de la estructura organizacional en el funcionamiento (Hao et al., 2012).

Tabla 2. Matriz de correlaciones de Pearson.

\begin{tabular}{|c|c|c|c|c|c|c|}
\hline & & $\begin{array}{l}\text { Empresas que } \\
\text { realizan innovación } \\
\text { de proceso y de } \\
\text { producto por región }\end{array}$ & $\begin{array}{l}\text { PIB } \\
\text { per cápita } \\
\text { regional } \\
2012\end{array}$ & $\begin{array}{c}\text { \% de gasto } \\
\text { en } \mathrm{I}+\mathrm{D} \\
\text { por región }\end{array}$ & $\begin{array}{l}\% \text { de gasto } \\
\text { en I+D } \\
\text { Educación } \\
\text { superior }\end{array}$ & $\begin{array}{c}\mathrm{N}^{\circ} \text { de empresas } \\
\text { que posee unidad } / \\
\text { depto. / laboratorio } \\
\text { de I+D }\end{array}$ \\
\hline \multirow{3}{*}{$\begin{array}{l}\text { Empresas que } \\
\text { realizan innovación } \\
\text { de proceso y de } \\
\text { producto por región }\end{array}$} & Correlación de Pearson & 1 & $0,962^{* *}$ & $0,973^{* *}$ & $0,875^{* *}$ & $0,959^{* *}$ \\
\hline & Sig. (bilateral) & & 0,000 & 0,000 & 0,000 & 0,000 \\
\hline & $\mathrm{N}$ & 15 & 15 & 15 & 15 & 15 \\
\hline \multirow{3}{*}{ PIB regional 2012} & Correlación de Pearson & $0,962^{* *}$ & 1 & $0,986^{* *}$ & $0,871^{* *}$ & $0,954^{* *}$ \\
\hline & Sig. (bilateral) & 0,000 & & 0,000 & 0,000 & 0,000 \\
\hline & $\mathrm{N}$ & 15 & 15 & 15 & 15 & 15 \\
\hline \multirow{3}{*}{$\begin{array}{l}\% \text { de gasto en } \mathrm{I}+\mathrm{D} \\
\text { por región }\end{array}$} & Correlación de Pearson & $0,973^{* *}$ & $0,986^{* *}$ & 1 & $0,872^{* *}$ & $0,964^{* *}$ \\
\hline & Sig. (bilateral) & 0,000 & 0,000 & & 0,000 & 0,000 \\
\hline & $\mathrm{N}$ & 15 & 15 & 15 & 15 & 15 \\
\hline \multirow{4}{*}{$\begin{array}{l}\% \text { de gasto en I+D } \\
\text { educación superior }\end{array}$} & Correlación de Pearson & $0,875^{\text {** }}$ & $0,871^{* *}$ & $0,872^{* *}$ & 1 & $0,815^{* *}$ \\
\hline & Sig. (bilateral) & 0,000 & 0,000 & 0,000 & & 0,000 \\
\hline & $\mathrm{N}$ & 15 & 15 & 15 & 15 & 15 \\
\hline & $\mathrm{N}$ & 15 & 15 & 15 & 15 & 15 \\
\hline \multirow{3}{*}{$\begin{array}{l}\text { Número de } \\
\text { empresas que posee } \\
\text { unidad/depto/ } \\
\text { laboratorio deI+D }\end{array}$} & Correlación de Pearson & $0,959^{* *}$ & $0,954^{* *}$ & $0,964^{* *}$ & $0,815^{* *}$ & 1 \\
\hline & Sig. (bilateral) & 0,000 & 0,000 & 0,000 & 0,000 & \\
\hline & $\mathrm{N}$ & 15 & 15 & 15 & 15 & 15 \\
\hline
\end{tabular}

** La correlación es significativa al nivel 0,01 (bilateral).

Fuente: Procesamiento de la información. 
Asimismo, es posible establecer que existe una alta correlación entre las variables independientes, razón por la que no se pudieron incorporar todas las variables para evitar problemas de multicolinealidad.

Continuando con los análisis multivariados, se puede establecer que el número de empresas que realizan innovación de proceso y de producto por región se ve determinado de manera significativa $(p<0,01)$ por el porcentaje de gasto en $I+D$ que ejecutan las instituciones de educación superior y por la presencia en estas organizaciones de unidades, departamentos o laboratorios de $\mathrm{I}+\mathrm{D}(\mathrm{R}=0,972$; $\mathrm{F}=134,101 ; \mathrm{p}<0,00)$. Por consiguiente, se puede apoyar la hipótesis formulada en la investigación.

Los resultados de la Tabla 3 indican que el porcentaje de gasto en I+D que se ejecuta por medio de las instituciones de educación superior de la región resulta determinante al momento de abordar y analizar las innovaciones, tanto de producto como de proceso, efectuadas por las empresas en las regiones de Chile $(\mathrm{p}<0,05)$. Esto es crucial, pues al momento de analizar los procesos de innovación se deben considerar los otros actores que forman parte de este y que influyen de manera significativa en dicho proceso, como sucede con estas instituciones. En relación con esto se puede destacar que las universidades son consideradas como un actor relevante y distintivo en los sistemas de innovación contribuyendo al potencial innovador de las sociedades, principalmente por su rol en la investigación básica caracterizada por sus altos niveles de tecnológicos (Veugelers et al., 2012). Por esta razón resulta fundamental la vinculación con el medio que desarrollan las instituciones de educación terciaria, la que en muchos casos forma parte de su misión (ver Tabla 3).

Del mismo modo, tiene vital importancia para la sociedad del conocimiento la formación de capital humano avanzado, con la docencia, la que constituye una de las funciones estratégicas de las universidades (Rodríguez et al., 2013). Particularmente hoy cuando la educación superior es un imperativo estratégico para las naciones, porque se configura como la base fundamental para la investigación, la creatividad y la innovación (Pedraja Rejas y Rodríguez Ponce, 2014).

Respecto del impacto que tiene sobre la innovación, tanto de producto como de proceso en las empresas, la presencia de unidades, departamentos o laboratorios de I+D en las compañías de las regiones de Chile, resulta altamente significativo $(p<0,00)$, debido a que una empresa que mantiene su propio departamento de investigación y desarrollo se puede considerar como un aspecto fundamental de diferenciación entre las firmas que tienen una conducta orientada efectivamente hacia la investigación y desarrollo de manera permanente, en comparación a otras que consideran estas actividades

Tabla 3. Resumen del modelo de regresión.

\begin{tabular}{lc}
\hline & $\begin{array}{c}\text { Número de empresas que realizan } \\
\text { innovación de proceso y de } \\
\text { producto por región }\end{array}$ \\
\hline $\mathrm{R}$ & 0,972 \\
$\mathrm{R}$ cuadrado & 0,946 \\
$\mathrm{R}$ cuadrado corregida & 0,937 \\
Error típ. de la estimación & 134,101 \\
$\mathrm{~F}$ & 104,560 \\
Sig. & $0,000 \mathrm{a}$ \\
Test t constante & 0,520 \\
Sig. Test T constante & 0,61 \\
Test t $\mathrm{N}^{\mathbf{o}}$ de empresas que posee unidad / depto. / laboratorio de I+D & 6,321 \\
Sig. Test t $\mathrm{N}^{\mathrm{o}}$ de empresas que posee unidad / depto. / laboratorio de I+D & 0,000 \\
Coeficiente beta de empresas que posee unidad / depto. / laboratorio de I+D & 0,733 \\
Test t \% de gasto en I+D que ejecutan las instituciones de educación superior & 2,386 \\
Sig. Test \% de gasto en I+D que ejecutan las instituciones de educación superior & 0,034 \\
Coeficiente beta \% de gasto en I+D que ejecutan las instituciones de educación superior & 0,277 \\
\hline
\end{tabular}

a Variables predictoras: (Constante), Liderazgo participativo y liderazgo colaborativo. Fuente: Procesamiento de la información. 
de manera ocasional (Czarnitzki et al., 2011). De acuerdo con esto último, se puede deducir además la relevancia que tiene para las regiones poseer capital humano avanzado y altamente calificado, siendo trascendental para liderar los procesos de innovación, así como también para poder absorber la tecnología y los conocimientos que se van demandando, siendo primordiales las instituciones de educación superior para proveer a las regiones de los profesionales calificados requeridos (PedrajaRejas y Rodríguez-Ponce 2013).

Ahora bien, en contraste con las grandes empresas, las pequeñas generalmente se comprometen en menores proyectos de innovación, ya que sus recursos y competencias son más limitados (Berends et al., 2014).

\section{Conclusiones}

Al momento de analizar el fenómeno de la innovación en las regiones de Chile se puede establecer que es un proceso sistémico, al involucrar a diversos actores locales en el proceso, como lo son las universidades, las empresas y las instituciones privadas sin fines de lucro. Además es multivariado, ya que son diversos los factores que repercuten en la innovación, tanto de proceso como de producto, que realizan las empresas analizadas.

En consecuencia, el número de empresas que realizan innovaciones de proceso y de producto en cada región se ve determinado, en primera instancia, por el porcentaje de gasto en investigación y desarrollo que es ejecutado por las instituciones de educación superior. Por tanto, las universidades juegan un importante papel no solo en la formación de capital humano, sino que también en la producción de conocimiento, los que al ser transferidos al medio van generando sinergias en el sistema regional de innovación, incrementando así el número de empresas dedicadas a la realización de innovaciones, fundamentales por lo demás para lograr el crecimiento económico del territorio.

Por último cabe mencionar el impacto positivo que tiene en los procesos de innovación la presencia de una unidad, departamento o laboratorio dedicado exclusivamente a las labores de investigación y desarrollo al interior de las empresas regionales, siendo fundamental para la continuidad y la celeridad que se le da a la innovación en el territorio.

\section{Literatura Citada}

Albors-Garrigós, J.; Rincon-Diaz, C.; Igartua-Lopez. J. 2014. Research technology organisations as leaders of R\&D collaboration with SMEs: role, barriers and facilitators. Technology Analysis \& Strategic Management, 26: 37-53.

Álvarez, R.; Bravo-Ortega, C.; Navarro, L.

2011. Innovación, investigación y desarrollo, y productividad en Chile. Revista CEPAL, 104: 141-166.

Archibugi, D.; Filippetti, A.; Frenz, M.

2013. Economic crisis and innovation: Is destruction prevailing over accumulation?". Research Policy, 42 (2): 303-314.

Bagautdinova, N.; Galieva, G.; Pakhmutov, Y.; Pratchenko, O. 2014. Methods of Regulation of Processes of Innovation Business Development. Mediterranean Journal of Social Sciences, 5 (12): 75.

Berends, H.; Jelinek, M.; Reymen, I.; Stultiëns, R.

2014. Product Innovation Processes in Small Firms: Combining entrepreneurial effectuation and managerial causation. Journal of Product Innovation Management, 31 (3): 616-635.

Cancino, $\mathrm{R}$.

2011. Complejidad y Evolución en Sistemas de Conocimiento: Dispositivos de producción de complejidad en el sistema científico-técnico en Chile. Revista Mad, 25: 51-62.

Capello. R.

2014. Proximity and regional innovation processes: is there space for new reflections? In: Torre, A.; Wallet, F. (Eds). Regional Development and Proximity Relations, pp. 163-194.
Carlsson, B.; Stankiewicz, R.

1991. On the nature, function and composition of technological systems". Journal of evolutionary economics, 1 (2): 93-118.

Carlsson, B.; Jacobsson, S.; Holmén, M.; Rickne, A.

2002. Innovation systems: analytical and methodological issues. Research policy. 31 (2): 233-245.

Cooke, $\mathrm{P}$.

2008. Regional Innovation Systems, Clean Technology \& Jacobian Cluster-Platform Policies. Regional Science Policy \& Practice, 1 (1): 23-45.

Cruz, A.

2008. La Ruta a la Innovación en Chile. Journal of Technology Management \& Innovation, 3 (1): 1-9.

Czarnitzki, D.; Hanel, P.; Rosa, J.

2011. Evaluating the impact of R\&D tax credits on innovation: A microeconometric study on Canadian firms. Research Policy, 40 (2): 217-229.

Dankbaar, B, Cobbenhagen, J.

2000. Learning and collaboration in practice. In Boekema, F.; Morgan, K.; Bakkers, S.; Rutten, R. (Eds.) . Knowledge, innovation and economic growth: The theory and practice of learning regions. London, Edward Elgar Publishing. 266 p.

Dodgson, M.; Hughes, A.; Foster, J.; Metcalfe. S.

2011. Systems thinking, market failure, and the development of innovation policy: The case of Australia. Research Policy, 40 (9): 1145-1156. 
Feldman, M.

2014. The character of innovative places: entrepreneurial strategy, economic development, and prosperity. Small Business Economics, 43 (1): 9-20.

Fernández de Lucio, I.; Vega Jurado, J.; Gutiérrez Gracia, A. 2011. Ciencia e innovación: una relación compleja y evolutiva. Arbor, 187: 1077-1089.

Filippetti, A.; Archibugi, D.

2011. Innovation in times of crisis: National Systems of Innovation, structure, and demand. Research Policy, 40 (2): $179-192$.

Freeman, C.

1988. Japan: a new national system of innovation. In: G. Dosi, C. Freeman, R. Nelson, G. Siverberg, L. Soete (Eds.), Technical Change and Economic Theory. Francis Pinter, London, pp. 330-348.

Galindo, M.; Méndez, M.

2014. Entrepreneurship, economic growth, and innovation: Are feedback effects at work? Journal of Business Research, 67(5): 825-829.

Gámir, L., Durá, P.

2010. La investigación y la innovación en España. Resultados y recursos en un marco comparado, Instituto de Estudios Económicos, Madrid. 264 p.

González, L.; Espinoza, O.

2011. La educación superior en Chile. Pensamiento Universitario, 22: 111-120.

Hajek, P.; Henriques, R.; Hajkova, V.

2014. Visualising components of regional innovation systems using self-organizing maps-Evidence from European regions. Technological Forecasting and Social Change, 84: 197-214.

Hao, Q.; Kasper, H.; Muehlbacher, J.

2012. How does organizational structure influence performance through learning and innovation in Austria and China. Chinese Management Studies, 6(1): 36-52.

Jacobsson, S.; Bergek, A.

2011. Innovation system analyses and sustainability transitions: Contributions and suggestions for research. Environmental Innovation and Societal Transitions, 1 (1): 41-57.

Malerba, F.

2005. Sectoral systems of innovation: a framework for linking innovation to the knowledge base, structure and dynamics of sectors. Economics of Innovation and New Technology, 14(1-2): 63-82.

Moreno, R.; Paci, R.; Usai, S.

2006. Innovation clusters in the European regions. European Planning Studies, 14 (9): 1235-1263.

Nelson, R.

1993. National innovation systems: a comparative analysis. University of Illinois at Urbana-Champaign's Academy for Entrepreneurial Leadership Historical Research Reference in Entrepreneurship. 560 p.

OECD, EUROSTAT.

2005. Manual de Oslo. Guía para la recogida e interpretación de datos sobre innovación. 190 p.
OECD.

2009. Policy Responses to the Economic Crisis: Investing in Innovation for Long-Term Growth. OECD, Paris. 37 p.

Padilla-Pérez, R.; Gaudin, Y.

2014. Science, technology and innovation policies in small and developing economies: The case of Central America. Research Policy, 43 (4): 749-759.

Páez, I.

2012. Capital humano, redes externas e innovación en la industria colombiana. Estudios Gerenciales, 28: 81-107.

Pedraja-Rejas, L.; Rodríguez-Ponce, E.

2014. Desafíos y roles de la educación superior. Ingeniare. Revista chilena de ingeniería, 22 (2): 156-157.

Pedraja-Rejas, Liliana M., Rodríguez-Ponce, Emilio.

2013. El desafío de la calidad en las universidades. Idesia, 31 (4): 3-11.

Piñero, A.; Rodríguez Monroy, C.; Arzola, M.

2013. Modelo de Política Pública de I+ D+ i, Para Liderar y Coordinar el Sistema Regional de Innovación en Apoyo a las PYMIS: Caso de Estudio del Estado de Bolívar, Venezuela. Journal of technology management \& innovation. 8: 34-34.

Prevezer, M.; Panzarasa, P.

2013. Regional innovation and performance: The role of absorptive capacity, industrial structure and collaborative networks in the Chinese provinces of Hubei and Hunan. Journal of Chinese Entrepreneurship, 5 (3): 196-219.

Radosevic, S., Kriaucioniene, M.

2014. Higher education in national innovation. In Bridges, D., Juceviciene, P., Jucevicius, R., Mclaughlin, T.H., \& Stankeviciute, J. (Eds.). Higher education and national development: Universities and societies in transition. Routledge. pp. 135-160.

Rodríguez-Ponce, E.; Pedraja-Rejas, L.; Araneda-Guirriman, C.; Rodríguez-Ponce, J.

2013. La relación entre la gestión del conocimiento y la gestión académica: un estudio exploratorio en universidades chilenas. Interciencia, 38 (2): 88-94.

Romer. P.

1990. Endogenous technological change. Journal of political Economy, 98 (5): 71-102.

Santillán. M.

2012. Innovación y los factores que la condicionan. Journal de Investigación de la Escuela de Graduados e Innovación, Agosto-Diciembre: 12-20.

Schumpeter, J.

1934. The theory of economic development: An inquiry into profits, capital, credit, interest, and the business cycle, Harvard University Press, 255 p.

Veugelers, R.; Callaert, J.; Song, X.; Van Looy, B.

2012. The participation of universities in technology development: do creation and use coincide? An empirical investigation on the level of national innovation systems. Economics of Innovation and New Technology, 21 (5-6): 445-472. 
